\title{
Rab15 expression correlates with retinoic acid-induced differentiation of neuroblastoma cells
}

\author{
NORIYUKI NISHIMURA ${ }^{1,2}$, THI VAN HUYEN PHAM ${ }^{1}$, TRI BUDI HARTOMO ${ }^{2}$, MYEONG JIN LEE $^{2}$, \\ DAIICHIRO HASEGAWA ${ }^{3}$, HIROKI TAKEDA ${ }^{3}$, KEIICHIRO KAWASAKI ${ }^{3}$, YOSHIYUKI KOSAKA ${ }^{3}$, \\ TOMOTO YAMAMOTO $^{1,2}$, SATORU MORIKAWA ${ }^{1,2}$, NOBUYUKI YAMAMOTO ${ }^{1}$, \\ IKUKO KUBOKAWA ${ }^{1}$, TAKESHI MORI ${ }^{1}$, TOMOKO YANAI ${ }^{1}$, AKIRA HAYAKAWA $^{1}$, \\ YASUHIRO TAKESHIMA ${ }^{1}$, HISAHIDE NISHIO ${ }^{1,2}$ and MASAFUMI MATSUO ${ }^{1}$ \\ Departments of ${ }^{1}$ Pediatrics and ${ }^{2}$ Epidemiology, Kobe University Graduate School of Medicine,
Kobe 650-0017; ${ }^{3}$ Department of Hematology and Oncology, Hyogo Children's Hospital, Kobe 654-0081, Japan
}

Received November 26, 2010; Accepted January 21, 2011

DOI: 10.3892/or.2011.1255

\begin{abstract}
Neuroblastoma is the most common extracranial solid tumor in children and accounts for $15 \%$ of pediatric cancer deaths. Although retinoic acid (RA) is currently used to treat high-risk neuroblastoma patients in the clinic, RA-responsiveness is variable and unpredictable. Since no alterations in the RA-signaling pathway have been found in neuroblastoma cells, molecules correlated with RA-induced differentiation will provide predictive markers of RA-responsiveness for clinical use. The Rab family of small $G$ proteins are key regulators of membrane traffic and play a critical role in cell differentiation and cancer progression. Although an increasing number of cancer-associated alternative splicing events have been identified, alternative splicing of Rab proteins remains to be characterized in neuroblastoma. In the present study, we focused on Rab15 that was originally identified as a brain-specific Rab protein and regulates the endocytic recycling pathway. We identified alternatively spliced Rab15 isoforms designated as Rab15 ${ }^{\mathrm{CN}}$ and Rab15 ${ }^{\mathrm{AN}}$ in neuroblastoma cells. Rab15 ${ }^{\mathrm{CN}}$ was composed of 7 exons encoding 212 amino acids and showed brain-specific expression. Alternative splicing of exon 4 generated Rab15 ${ }^{\mathrm{AN}}$ that was predicted to encode 208 amino acids and was predominantly expressed in testis. RA induced neuronal differentiation of neuroblastoma $\mathrm{BE}(2)-\mathrm{C}$ cells and specifically up-regulated Rab15 ${ }^{\mathrm{CN}}$ expression. Reciprocally, RA-induced differentiation was observed in Rab15 ${ }^{\mathrm{CN}}$-expressing $\mathrm{BE}(2)-\mathrm{C}$ cells in preference to Rab15 ${ }^{\mathrm{AN}}$-expressing BE(2)-C cells. Furthermore, Rab15 ${ }^{\mathrm{CN}}$ expression was also specifically up-regulated during RA-induced differentiation of newly established neuroblas-
\end{abstract}

Correspondence to: Dr Noriyuki Nishimura, Departments of Pediatrics and Epidemiology, Kobe University Graduate School of Medicine, 7-5-1 Kusunoki-cho, Chuo-ku, Kobe 650-0017, Japan E-mail: nnishi@med.kobe-u.ac.jp

Key words: Rab15, alternative splicing, retinoic acid, neuroblastoma toma cells from high-risk patients. These results suggest that Rab15 expression correlates with RA-induced differentiation of neuroblastoma cells.

\section{Introduction}

Neuroblastoma is the most common extracranial solid tumor in children and accounts for $15 \%$ of pediatric cancer deaths. More than $50 \%$ of high-risk neuroblastoma patients develop early or late relapse (1-3). This is mainly due to the difficulty in eradicating the minimal residual disease (MRD) that resides in the bone marrow and peripheral blood even after achieving the complete remission. With the aim of inducing the differentiation of MRD, the vitamin A metabolite retinoic acid (RA) is currently used in the clinic for high-risk neuroblastoma patients. Although RA can let neuroblastoma cells cease proliferation, differentiate into neuronal cells or undergo apoptosis, RA-responsiveness of high-risk neuroblastoma patients is variable and unpredictable. Consequently, less than $40 \%$ of high-risk neuroblastoma patients can expect a long-term cure $(4,5)$. Since no alteration in the RA-signaling pathway is found in neuroblastoma cells, a molecule correlated with RA-induced differentiation will give insight into a predictive marker of RA-responsiveness for clinical use.

Phenotypic characterization of a large number of neuroblastoma cells identifies three major cell types, designated as $\mathrm{N}$ (neuroblastic), S (substrate-adherent and non-neuronal) and I (intermediate) (6,7). N-type neuroblastoma cells have small and rounded cell bodies with neurite-like processes and attach poorly to the substrate. In contrast, S-type cells have large and flattened cell bodies without neurite-like processes and grow in culture as an adherent monolayer. I-type cells have an intermediate morphology between N-type and S-type, and can differentiate into either $\mathrm{N}$-type or S-type when induced by specific agents. BE(2)-C cells have a typical I-type phenotype and are frequently used as a model for RA-induced differentiation of neuroblastoma cells (8).

Rab family small $G$ proteins are central in ensuring the spatiotemporal regulation of membrane traffic. Over 60 
Table I. Summary of clinical samples.

\begin{tabular}{lclcc}
\hline Sample name & Age (years) & Gender & Tumor risk group & Specimen type \\
\hline NBBM2 & 6 & Male & High & Bone marrow \\
NBBM3 & 3 & Female & High & Bone marrow \\
NBTT2D & 2 & Male & High & Adrenal gland \\
\hline
\end{tabular}

Tumor risk group was based on the Children's Oncology Group Neuroblastoma Risk Stratification System (30).

different Rab proteins are identified in mammalian cells, and each Rab protein recognizes distinct subsets of intracellular membranes and regulates the specific membrane transport pathway (9-11). Accumulating evidence reveals that aberrant expressions of several Rab proteins are associated with cancer progression (12). Increased expression of brainspecific Rab3A and Rab23 is implicated in brain tumor, neuroendocrine tumors, hepatocellular carcinoma and gastric cancer (13-15). Rab25 and its effector Rab-coupling protein $(\mathrm{RCP})$ control the endocytic recycling pathway and are identified as drivers of genomic amplicon in ovarian and breast cancer $(16,17)$.

Alternative splicing represents an important molecular mechanism of gene regulation in normal cell growth and differentiation. In the case of hyaluronan synthase 1 (HAS1) and receptor for hyaluronan-mediated motility (RHAMM), their aberrant splicing are associated with multiple myeloma, and their isoform balance is shown to be a prognostic marker $(18,19)$. Although an increasing number of cancer-associated alternative splicing events are identified, alternative splicing of Rab proteins remains to be characterized in neuroblastoma $(20,21)$.

In the present study, we focused on Rab15 that was originally identified as a brain-specific Rab protein and regulated the endocytic recycling pathway $(22,23)$. We identified and characterized alternatively spliced Rab15 isoforms in neuroblastoma cells.

\section{Materials and methods}

Cell culture, transfection and neuronal differentiation. $\mathrm{BE}(2)-\mathrm{C}$ cells were obtained from ATCC (Manassas, VA), cultured at $37^{\circ} \mathrm{C}\left(5 \% \mathrm{CO}_{2}\right.$ and $95 \%$ air $)$ in Dulbecco's modified Eagle's medium (DMEM)/Ham's F12 (Wako Pure Chemical, Osaka, Japan) with $10 \%$ fetal bovine serum (FBS; Invitrogen, Carlsbad, CA), and transfected using Lipofectamine 2000 transfection reagent (Invitrogen) according to the manufacturer's instructions. For neuronal differentiation, BE(2)-C cells were treated with either dimethyl sulfoxide (DMSO; Wako Pure Chemical) or $10 \mu \mathrm{M}$ all-trans-retinoic acid (RA; Sigma, St. Louis, MO) for 3-7 days.

Quantitative real-time RT-PCR. Human tissue total RNA was purchased from Clontech (Palo Alto, CA). Total RNA from neuroblastoma cells was isolated with RNeasy Mini kit (Qiagen, Valencia, CA) and reverse transcribed using Quantitect Reverse Transcription kit (Qiagen). Real-time PCR analysis was performed with an ABI 7500 Fast real-time PCR
System (Applied Biosystems, Foster City, CA) using FastStart Universal SYBR-Green Master (Roche) according to the manufacturer's instructions. Each sample was analyzed in triplicate. Relative mRNA expression of Rab15 to PGK1 and Rab15 isoform balance were calculated by the comparative $\mathrm{C}_{\mathrm{T}}$ method. Primers for Rab15 ${ }^{\mathrm{CN}}$ (ENST00000436278) were 5'-AGAGATACCAGACCATCACA-3' (sense) and 5'-T TCTGGTGCGTACTCATCCACGTC-3' (anti-sense), Rab15 AN (ENST00000267512) were 5'-TACAGATCTGGGACACTGCA-3' (sense) and 5'-CCGGCAGTGAGGTGGCATCT-3' (anti-sense), and phosphoglycerate kinase 1 (PGK1, NM_000291) were 5'-GGAGAACCTCCGCTTTCAT-3' (sense) and 5'-GCTG GCTCGGCTTTAACC-3' (anti-sense).

Plasmid construction. The full-length cDNAs of human Rab15 CN (ENST00000436278) and Rab15 AN (ENST 00000267512) were cloned by RT-PCR from BE(2)-C cell cDNA, and cloned into the bicistronic expression vector pCMV6-AC-IRES-GFP (OriGene, Rockville, MD). The resulting pCMV6-Rab15 ${ }^{\mathrm{CN}}$-IRES-GFP and pCMV6-Rab15 ${ }^{\mathrm{AN}}$ IRES-GFP plasmids were sequenced using an ABI Prism 3100 Genetic Analyzer (Applied Biosystems).

Phase-contrast and immunofluorescence microscopy. Phase-contrast images of neuroblastoma cells were acquired using a BZ-9000E fluorescence microscope (Keyence, Osaka, Japan). Neuroblastoma cells grown on Collagen I-coated cover glass (Iwaki, Tokyo, Japan) were fixed with $2 \%$ formaldehyde in PBS for $15 \mathrm{~min}$ at room temperature. After permeabilization with $0.1 \%$ Triton X-100 in PBS for 5 min and blocking with $5 \%$ goat serum (Sigma) in PBS (GS/PBS) for $60 \mathrm{~min}$ at room temperature, cells were incubated with primary antibodies in GS/PBS for $60 \mathrm{~min}$, with Alexa 568-conjugated secondary antibodies (Invitrogen) in GS/PBS for $60 \mathrm{~min}$, and with $1 \mu \mathrm{g} / \mathrm{ml} \mathrm{4,6-diamino-}$ 2-phenylindole (DAPI; Sigma) in PBS for $5 \mathrm{~min}$ at room temperature. Fluorescent images were acquired using a BZ-9000E fluorescence microscope (Keyence).

Newly established neuroblastoma cells from high-risk patients. Bone marrow and tumor samples were obtained from patients with high-risk neuroblastoma who were admitted to Hyogo Children's Hospital and gave the written informed consent approved by Hyogo Children's Hospital Ethics Board. These samples were handled in accordance with the Guidelines for Clinical Research of Kobe University Graduate School of Medicine. Bone marrow samples were 
A

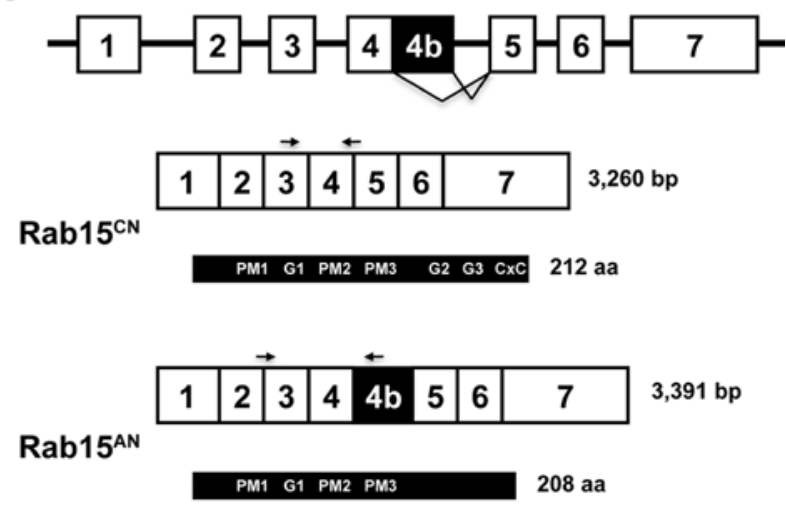

B

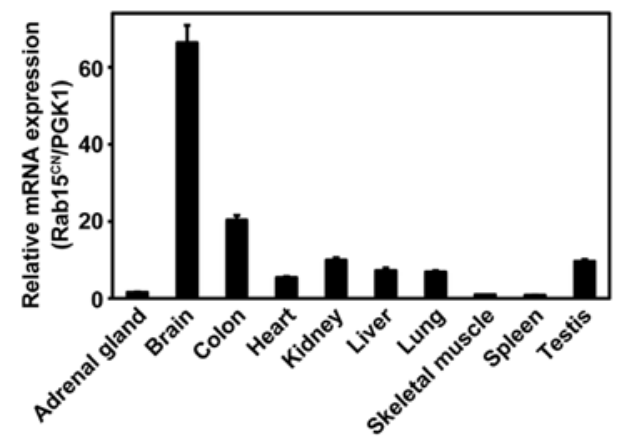

C

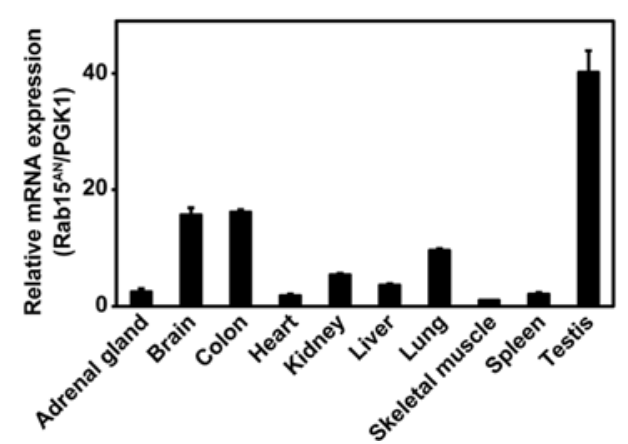

Figure 1. Identification and tissue distribution of alternatively spliced Rab15 isoforms. (A) The structure of Rab15 gene, Rab15 ${ }^{\mathrm{CN}}$ and Rab15 $\mathrm{AN}$ PM1-PM3, G1-G3, and CxC are the conserved residues for magnesium/ phosphate-binding, GDP/GTP-binding, and geranylgeranylation in Rab proteins, respectively (24). Arrows indicate the primers used for a real-time RT-PCR. The sequences of primers are given in Materials and methods. (B and C) The relative mRNA expression of Rab15 ${ }^{\mathrm{CN}}$ to PGK1 (B) and Rab15 to PGK1 (C) in various human tissues was analyzed by quantitative realtime RT-PCR. The mean expression in skeletal muscle was set to 1 . The data shown are the means \pm SD of three independent experiments.

separated by Mono-Poly Resolving Medium (DS Pharma Biomedical, Osaka, Japan) and mono- and poly-nucleated cells were collected. The resulting bone marrow cells were washed with $\mathrm{PBS}$, cultured at $37^{\circ} \mathrm{C}\left(5 \% \mathrm{CO}_{2}\right.$ and $95 \%$ air $)$ in DMEM/Ham's F12 containing 10\% FBS, and designated as NBBM2 and NBBM3 cells (Table I). Tumor tissue samples were collected, cut into $2-3 \mathrm{~mm}^{3}$ pieces, enzymatically dissociated with Liberase DH (Roche, Mannheim, Germany) in PBS for $15-45 \mathrm{~min}$ at $37^{\circ} \mathrm{C}$ followed by the addition of $10 \%$ FBS to inhibit enzyme activity, and filtered through a $100-\mu \mathrm{m}$ cell strainer (BD, Bedford, MA). The resulting tumor tissue cells were washed with $\mathrm{PBS}$, cultured at $37^{\circ} \mathrm{C}$ (5\% $\mathrm{CO}_{2}$ and $95 \%$ air) in DMEM/Ham's F12 containing $10 \%$ FBS, and designated as NBTT2D cells (Table I).

Antibodies and other reagents. Mouse anti- $\beta$-1, 3-glucuronyltransferase 1 (B3GAT1)/CD57/HNK-1 antibody was purchased from $\mathrm{BD}$ and mouse anti-tyrosine hydroxylase $(\mathrm{TH})$ from Millipore (Temecula, CA). Other reagents were obtained from commercial sources.

\section{Results}

Identification and tissue distribution of alternatively spliced Rab15 isoforms. Rab15 cDNA was originally isolated from a rat brain cDNA library and contained a 639 bp open reading frame encoding 212 amino acids (22). In human, Rab15 gene is composed of 7 exons and predicted to have an alternatively spliced exon 4 (Fig. 1A). We first cloned the full-length cDNAs for two human Rab15 transcripts (ENST00000436278 and ENST00000267512) by RT-PCR from neuroblastoma $\mathrm{BE}(2)-\mathrm{C}$ cells and confirmed their DNA sequences. Here, we designated ENST00000436278 and ENST00000267512 as canonical Rab15 (Rab15 ${ }^{\mathrm{CN}}$ ) and alternative Rab15 ( $R a b 15^{\mathrm{AN}}$ ), respectively. Rab15 ${ }^{\mathrm{CN}}$ was a human counterpart of rat Rab15 and contained a 78-bp exon 4, resulting in a 639 bp open reading frame encoding 212 amino acids. Rab15 ${ }^{\text {AN }}$ was an alternatively spliced isoform of human Rab15 and had a 131-bp insertion (exon $4 b$ ) between exon 4 and exon 5, resulting in a 627 bp open reading frame encoding 208 amino acids (Fig. 1A). Amino acid sequence analysis revealed that exon $4 \mathrm{~b}$ insertion resulted in a frameshift in Rab15 ${ }^{\mathrm{AN}}$ and generated a unique Carboxy-terminal tail of 100 amino acids lacking the conserved residues for GDP/GTP-binding (G2 and G3) and geranylgeranylation $(\mathrm{CxC})$ in $\mathrm{Rab}$ proteins (24). Next we examined the expression of Rab15 ${ }^{\mathrm{CN}}$ and Rab15 $5^{\mathrm{AN}}$ in various human tissues by a quantitative real-time RT-PCR using the Rab15 isoform-specific primers (Fig. 1A). In accordance with previous report (22), Rab15 ${ }^{\mathrm{CN}}$ mRNA was highly expressed in brain, and at low levels in other tissues and testis (Fig. 1B). In contrast, testis showed the highest level of Rab15 ${ }^{\mathrm{AN}}$ expression, and other tissues and brain showed only slight expression (Fig. 1C).

Differential expression of Rab15 ${ }^{C N}$ and Rab15 ${ }^{A N}$ during $R A$-induced differentiation of BE(2)-C cells. As reported previously (8), BE(2)-C cells responded to RA and extended neurite-like processes (Fig. 2A). We then analyzed the expression of Rab15 ${ }^{\mathrm{CN}}$ and Rab15 ${ }^{\mathrm{AN}}$ in DMSO-treated and $\mathrm{RA}$-treated $\mathrm{BE}(2)-\mathrm{C}$ cells. In response to $\mathrm{RA}$, the relative expression of Rab15 ${ }^{\mathrm{CN}}$ and Rab15 ${ }^{\mathrm{AN}}$ to PGK1 was increased up to 2.1- and 1.1-fold, respectively (Fig. 2B). These results suggest that RA induces the differential expression of Rab15 ${ }^{\mathrm{CN}}$ and Rab15 ${ }^{\mathrm{AN}}$ in $\mathrm{BE}(2)-\mathrm{C}$ cells.

Distinct RA-responsiveness of Rab15 ${ }^{C N}$-expressed and Rab15 ${ }^{A N}$-expressed BE(2)-C cells. To gain insight into the function of Rab15 $\mathrm{CN}$ and Rab15 $5^{\mathrm{AN}}$, we then examined the effect of Rab15 ${ }^{\mathrm{CN}}$ and Rab15 $\mathrm{AN}$ expression on the RA-induced 
A

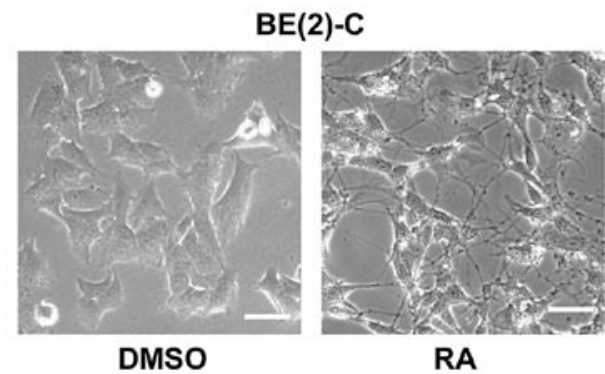

B

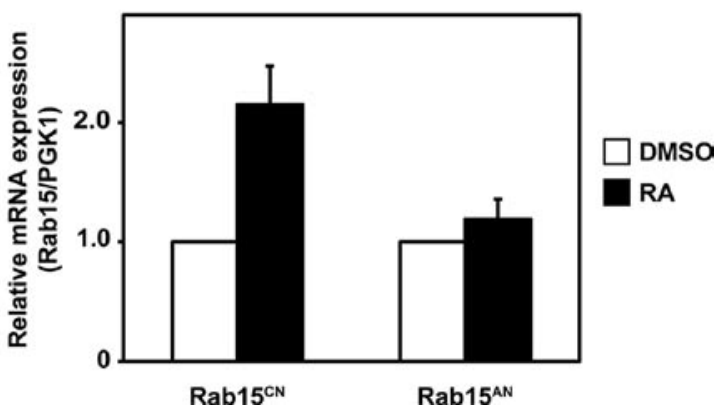

Figure 2. Differential expression of Rab15 ${ }^{\mathrm{CN}}$ and Rab15 $5^{\mathrm{AN}}$ during RA-induced differentiation of $\mathrm{BE}(2)-\mathrm{C}$ cells. (A) $\mathrm{BE}(2)-\mathrm{C}$ cells were treated with either DMSO or $10 \mu \mathrm{M}$ RA for 7 days and examined by phase-contrast microscopy. The images shown are representative of three independent experiments. Scale bars, $50 \mu \mathrm{m}$. (B) Total RNA was prepared from DMSO-treated and RA-treated BE(2)-C cells (DMSO and RA). The relative mRNA expression of Rab15 ${ }^{\mathrm{CN}}$ to PGK1 and Rab15 ${ }^{\mathrm{AN}}$ to PGK1 was analyzed by quantitative real-time RT-PCR. The mean expression in DMSO was set to 1 . The data shown are the means \pm SD of three independent experiments.

differentiation of $\mathrm{BE}(2)-\mathrm{C}$ cells. When Rab15 ${ }^{\mathrm{CN}}$-IRES-GFP

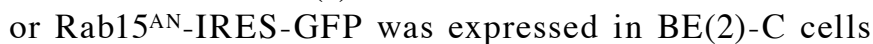
without RA, no morphological change was observed between GFP-positive and GFP-negative cells (Fig. 3). In response to
RA, Rab15 ${ }^{\mathrm{CN}}$-expressed cells extended neurite-like processes, while Rab15 ${ }^{\mathrm{AN}}$-expressed cells did not change their morphology (Fig. 3). These results implicate that the expression of Rab15 ${ }^{\mathrm{CN}}$ and Rab15 ${ }^{\mathrm{AN}}$ induces distinct RA-responsiveness in $\mathrm{BE}(2)-\mathrm{C}$ cells.

Characterization of newly established neuroblastoma cells from high-risk patients. Because neuroblastoma cells displayed considerable heterogeneity in response to RA (25), we have established three neuroblastoma cells designated as NBBM2, NBBM3, and NBTT2D from high-risk patients (Table I). The morphology of NBBM2 and NBTT2D cells were characterized by a round and flattened cell body with little neurite-like processes, and closely resembled I-type BE(2)-C cells (Figs. 2A and 5A). In contrast, NBBM3 cells displayed a spindle-shaped cell body with longer neurite-like processes that is typical for N-type neuroblastoma cells (Fig. 5A). To confirm the origin of NBBM2, NBBM3, and NBTT2D cells, we determined whether these cells indeed expressed neuroblastoma markers, $\beta$-1, 3-glucuronyltransferase 1 (B3GAT1, also known as CD57 and HNK-1) and tyrosine hydroxylase ( $\mathrm{TH})$, by an immunofluorescence microscopy. B3GAT1 is a marker for histologically immature neuroblastoma cells and neural crest stem cells (26), while TH is a catecholamine biosynthetic pathway enzyme used as a clinical neuroblastoma marker (27). Although the percentage of positive cells was varied, NBBM2, NBBM3, and NBTT2D cells indeed expressed both B3GAT1 and TH (Fig. 4).

Differential expression of Rab15 ${ }^{C N}$ and Rab15 ${ }^{A N}$ during RA-induced differentiation of newly established neuroblastoma cells from high-risk patients. To characterize newly established neuroblastoma cells, we first examined their RA-responsiveness. I-type NBBM2 and NBTT2D cells extended neurite-like processes and displayed a typical N-type morphology in response to RA (Fig. 5A). N-type NBBM3 cells were also responded to RA and elongated the length of neurite-like processes (Fig. 5A). Next, we analyzed the
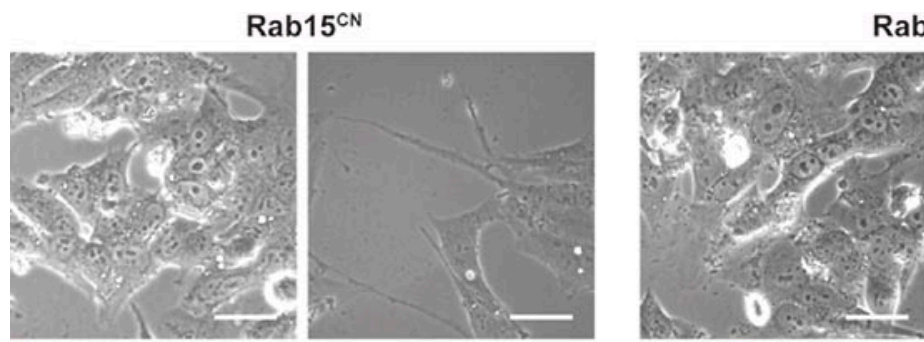

Rab15 ${ }^{\mathrm{AN}}$

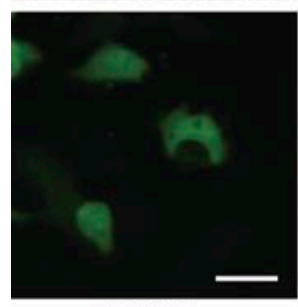

DMSO

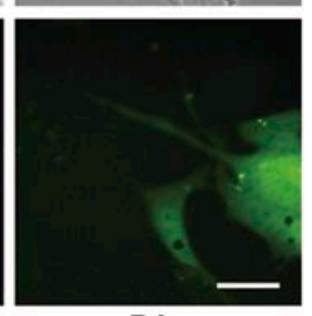

RA

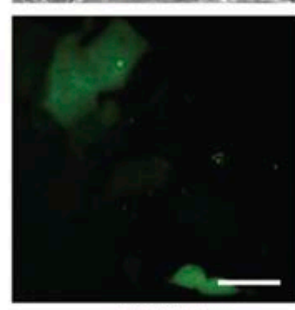

DMSO

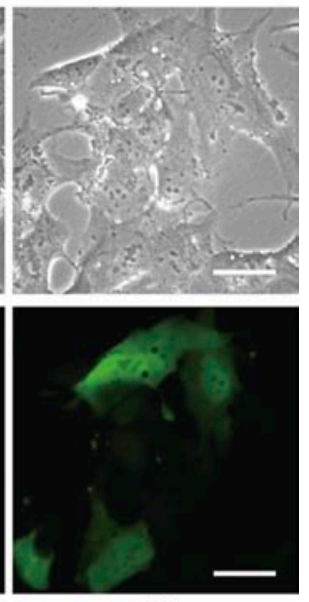

RA

Figure 3. Distinct RA-responsiveness of Rab15 ${ }^{\mathrm{CN}}$-expressed and Rab15 $5^{\mathrm{AN}}$-expressed BE(2)-C cells. BE(2)-C cells transfected with pCMV6-Rab15 ${ }^{\mathrm{CN}}$ IRES-GFP or pCMV6-Rab15 ${ }^{\mathrm{AN}}$-IRES-GFP were treated with DMSO or $10 \mu \mathrm{M}$ RA for 3 days and examined by phase-contrast and immunofluorescence microscopy. The images shown are representative of three independent experiments. Scale bars, $50 \mu \mathrm{m}$. 

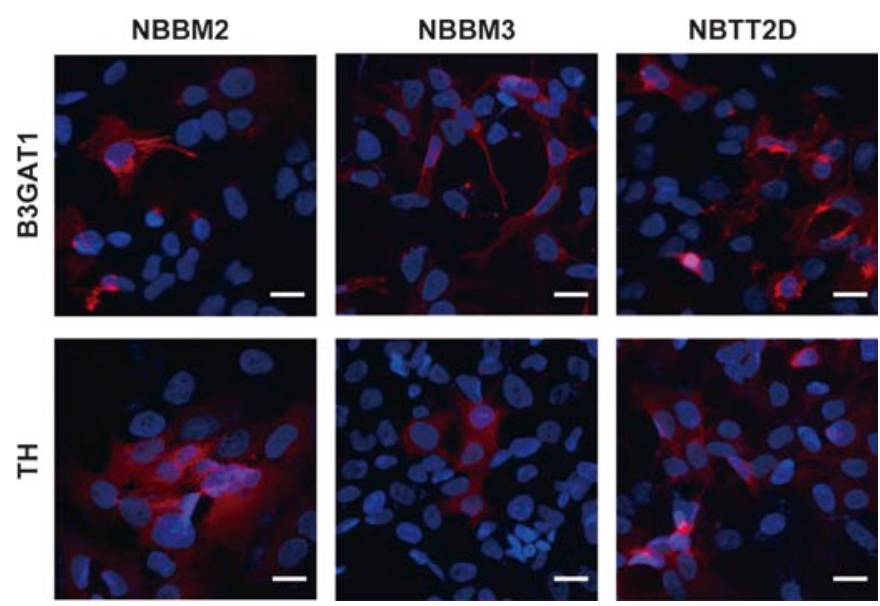

Figure 4. Characterization of newly established neuroblastoma cells from high-risk patients. NBBM2, NBBM3, and NBTT2D neuroblastoma cells were established from high-risk patients and immunostained with antiB3GAT1 or anti-TH antibody (Red). Nuclei were stained with DAPI (Blue). The images shown are representative of three independent experiments. Scale bars, $20 \mu \mathrm{m}$.

expression of Rab15 ${ }^{\mathrm{CN}}$ and $\mathrm{Rab} 15^{\mathrm{AN}}$ by a quantitative real-time RT-PCR using the Rab15 isoform-specific primers. Upon RA-treatment, Rab15 $\mathrm{CN}$ expression was increased up to 1.9-, 1.8-, and 2.0-fold in NBBM2, NBBM3, and NBTT2D cells, respectively (Fig. 5B). In contrast, Rab15 ${ }^{\mathrm{AN}}$ expression in NBBM2, NBBM3, and NBTT2D cells was changed 1.3-, 1.4-, and 1.2-fold, respectively (Fig. 5C). These results indicate that RA induces differential expression of Rab15 $\mathrm{CN}$ and Rab15 $\mathrm{AN}$ in NBBM2, NBBM3, and NBTT2D cells as well as BE(2)-C cells.

Correlation between Rab15 expression and RA-induced differentiation of neuroblastoma cells. As the isoform balance of some aberrantly spliced genes in cancer cells was reported to be a prognostic marker $(18,19)$, we first determined Rab15 isoform balance measured by the Rab15 CN/ Rab15 ${ }^{\mathrm{AN}}$ ratio in various human tissues. It was considerably varied, ranging from 0.6 in testis to 11.5 in brain (Fig. 6A). We then determined Rab15 isoform balance in BE(2)-C, NBBM2, NBBM3, and NBTT2D cells treated with DMSO and RA. It was significantly increased upon RA-treatment in all neuroblastoma cells (Fig. 6B). These results suggest that Rab15 expression correlates with RA-induced differentiation of neuroblastoma cells.

\section{Discussion}

In the present study, we have identified alternatively spliced Rab15 isoforms designated as Rab15 ${ }^{\mathrm{CN}}$ and Rab15 ${ }^{\mathrm{AN}}$ in neuroblastoma cells and obtained two novel findings. First, Rab15 ${ }^{\mathrm{AN}}$ is predominantly expressed in testis, while Rab15 $\mathrm{CN}$ shows a brain-specific expression. Second, Rab15 expression correlates with RA-induced differentiation of neuroblastoma cells.

Neuroblastoma cells are widely assumed to originate from neural crest cells that had a cancerous change during their sympathoadrenal differentiation (1-3). In response to $\mathrm{RA}$, neuroblastoma cells can differentiate into neuronal

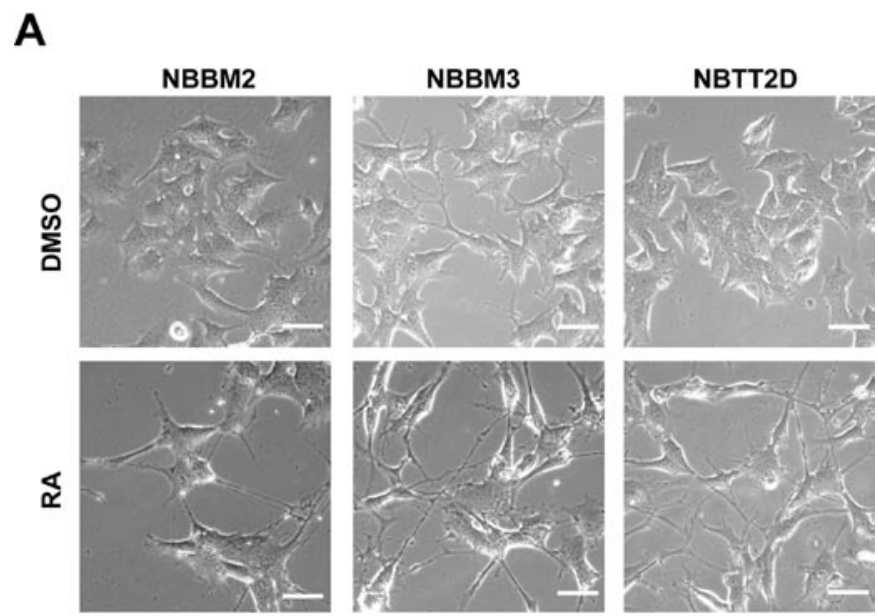

\section{B}

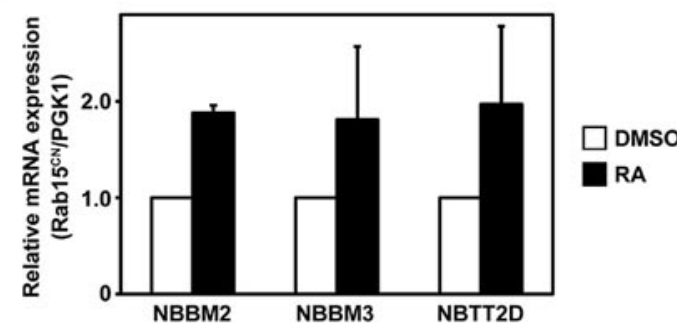

C

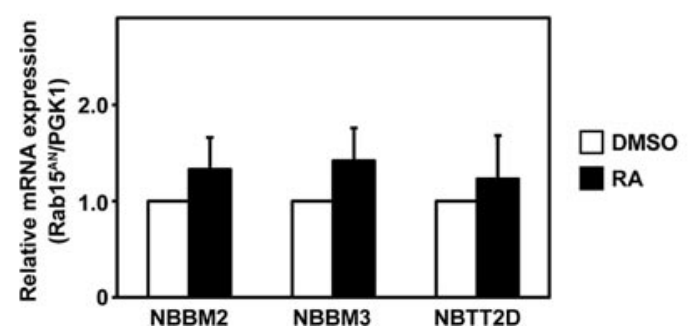

Figure 5. Differential expression of Rab15 ${ }^{\mathrm{CN}}$ and Rab15 $5^{\mathrm{AN}}$ during RA-induced differentiation of newly established neuroblastoma cells from high-risk patients. (A) NBBM2, NBBM3, and NBTT2D cells were treated with either DMSO or $10 \mu \mathrm{M}$ RA for 7 days and examined by phase-contrast microscopy. The images shown are representative of three independent experiments. Scale bars, $50 \mu \mathrm{m}$. (B and C) Total RNA was prepared from DMSO-treated NBBM2, NBBM3, and NBTT2D cells (DMSO) and RA-treated NBBM2, NBBM3, and NBTT2D cells (RA). The relative mRNA expression of Rab15 ${ }^{\mathrm{CN}}$ to PGK1 (B) and Rab15 ${ }^{\mathrm{AN}}$ to PGK1 (C) was analyzed by quantitative real-time RT-PCR. The mean expression in DMSO was set to 1 . The data shown are the means \pm SD of three independent experiments.

cells or undergo apoptosis. Although the heterogeneity in RA-responsiveness is widely recognized (25), all neuroblastoma cells treated with RA in the present study extended neuron-like processes and up-regulated Rab15 ${ }^{\mathrm{CN}}$ expression (Fig. 5). Rab15 $\mathrm{CN}$ expression in $\mathrm{BE}(2)-\mathrm{C}$ cells was not sufficient to induce the extension of neuron-like processes (Fig. 3). Although Rab15 up-regulation likely corresponds to the increased demand for the endocytic recycling pathway to remodel cell membranes and induce neuronal differentiation (23), the exact way by which Rab15 up-regulation could affect the RA-induced differentiation is an open question for future study. 
A

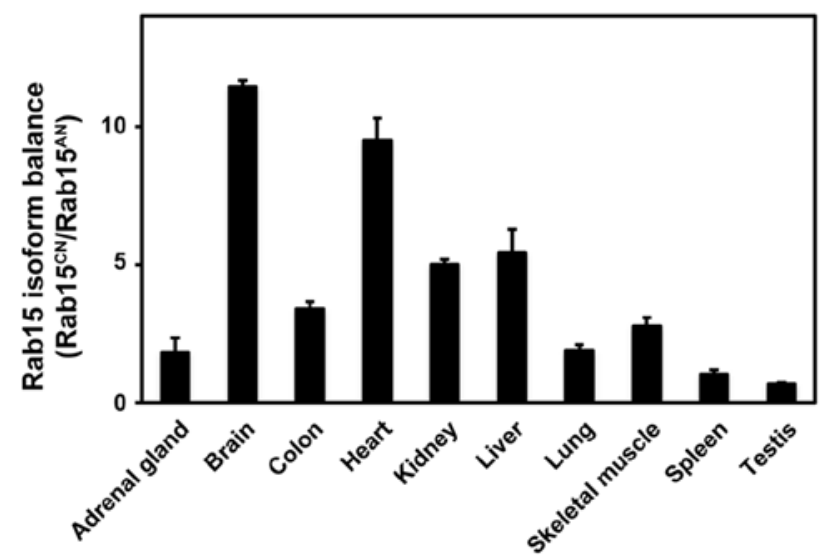

B

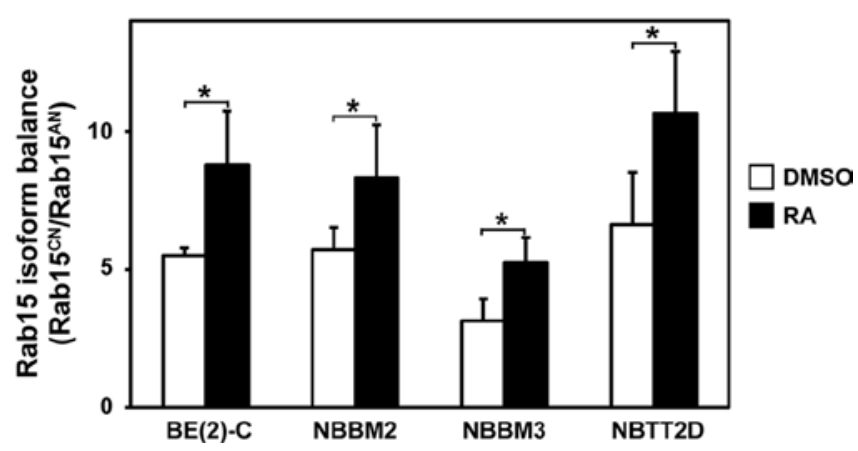

Figure 6. Correlation between Rab15 expression and RA-induced differentiation of neuroblastoma cells. Rab15 isoform balance measured by the Rab15 $\mathrm{AN} / \mathrm{Rab} 15^{\mathrm{CN}}$ ratio was analyzed by quantitative real-time RT-PCR in various human tissues (A) and in neuroblastoma cells (B). The data shown are means \pm SD of three independent experiments. Asterisk shows a significant difference (Student's t test, $\mathrm{P}<0.05$ ).

An increasing number of genes including HAS1 and RHAMM are shown to predict clinical outcome depending on the differential expression of alternatively spliced isoforms in cancer patients (18-21). Although alternative splicing events of Rab proteins remain to be characterized in neuroblastoma, Rab6A and Rab28 are reported to contain alternatively spliced isoforms. Rab6A isoforms named Rab6A and Rab6A' are ubiquitously expressed at similar levels, whereas Rab28 isoforms named Rab28S and Rab28L show differential expression: ubiquitous Rab28S and testis-specific Rab28L $(28,29)$. In the case of Rab15, it is not clear whether alternatively spliced isoform Rab15 $5^{\mathrm{AN}}$ has actual cellular function, or whether Rab15 ${ }^{\mathrm{AN}}$ is a consequence of aberrant mRNA splicing. We are now trying to elucidate the exact function of Rab15 ${ }^{\mathrm{AN}} \mathrm{mRNA}$ and its encoded protein in neuroblastoma cells. As RA-responsiveness of MRD in high-risk neuroblastoma patients is variable and unpredictable, Rab15 expression will give insight into a predictive marker of RA-responsiveness for clinical use $(4,5)$.

In summary, we identified alternatively spliced Rab15 isoforms, brain-specific Rab15 ${ }^{\mathrm{CN}}$ and testis-specific Rab15 ${ }^{\mathrm{AN}}$, in neuroblastoma cells, and we revealed that Rab15 expression correlated with RA-induced differentiation of neuroblastoma cells.

\section{Acknowledgements}

This study was supported in part by Grants-in-Aid for Scientific Research from the Ministry of Education, Culture, Sports, Science and Technology of Japan.

\section{References}

1. Janoueix-Lerosey I, Schleiermacher G and Delattre O: Molecular pathogenesis of peripheral neuroblastic tumors. Oncogene 29: 1566-1579, 2010.

2. Maris JM,Hogarty MD, Bagatell R and Cohn SL: Neuroblastoma. Lancet 369: 2106-2120, 2007.

3. Brodeur GM: Neuroblastoma: biological insights into a clinical enigma. Nat Rev Cancer 3: 203-216, 2003.

4. Reynolds CP, Matthay KK, Villablanca JG and Maurer BJ: Retinoid therapy of high-risk neuroblastoma. Cancer Lett 197: 185-192, 2003.

5. Wagner LM and Danks MK: New therapeutic targets for the treatment of high-risk neuroblastoma. J Cell Biochem 107: 46-57, 2009.

6. Ciccarone V, Spengler BA, Meyers MB, Biedler JL and Ross RA: Phenotypic diversification in human neuroblastoma cells: expression of distinct neural crest lineages. Cancer Res 49: 219-225, 1989.

7. Ross RA, Biedler JL and Spengler BA: A role for distinct cell types in determining malignancy in human neuroblastoma cell lines and tumors. Cancer Lett 197: 35-39, 2003.

8. Ross RA, Spengler BA, Domènech C, Porubcin M, Rettig WJ and Biedler JL: Human neuroblastoma I-type cells are malignant neural crest stem cells. Cell Growth Differ 6: 449-456, 1995.

9. Takai Y, Sasaki T and Matozaki T: Small GTP-binding proteins. Physiol Rev 81: 153-208, 2001.

10. Nishimura $\mathrm{N}$ and Sasaki $\mathrm{T}$ : Rab family small G proteins in regulation of epithelial apical junctions. Front Biosci 14: 2115-2129, 2009.

11. Stenmark H: Rab GTPases as coordinators of vesicle traffic. Nat Rev Mol Cell Biol 10: 513-525, 2009.

12. Chia WJ and Tang BL: Emerging roles for Rab family GTPases in human cancer. Biochim Biophys Acta 1795: 110-116, 2009.

13. Culine S, Rousseau-Merck MF, Honoré N, Nezelof C and Olofsson B: Specific expression of the ras-related rab3A gene in human normal and malignant neuroendocrine cells. Cancer 70: 2552-2556, 1992.

14. Liu Y-J, Wang Q, Li W, et al: Rab23 is a potential biological target for treating hepatocellular carcinoma. World $\mathrm{J}$ Gastroenterol 13: 1010-1017, 2007.

15. Hou Q, Wu YH, Grabsch H, et al: Integrative genomics identifies RAB23 as an invasion mediator gene in diffuse-type gastric cancer. Cancer Res 68: 4623-4630, 2008.

16. Cheng KW, Lahad JP, Kuo W, et al: The RAB25 small GTPase determines aggressiveness of ovarian and breast cancers. Nat Med 10: 1251-1256, 2004.

17. Zhang J, Liu X, Datta A, et al: RCP is a human breast cancerpromoting gene with Ras-activating function. J Clin Invest 119: 2171-2183, 2009.

18. Maxwell CA, Rasmussen E, Zhan F, et al: RHAMM expression and isoform balance predict aggressive disease and poor survival in multiple myeloma. Blood 104: 1151-1158, 2004.

19. Adamia S, Reiman T, Crainie M, Mant MJ, Belch AR and Pilarski LM: Intronic splicing of hyaluronan synthase 1 (HAS1): a biologically relevant indicator of poor outcome in multiple myeloma. Blood 105: 4836-4844, 2005.

20. Venables JP: Unbalanced alternative splicing and its significance in cancer. Bioessays 28: 378-386, 2006.

21. Ward AJ and Cooper TA: The pathobiology of splicing. J Pathol 220: 152-163, 2010.

22. Elferink LA, Anzai K and Scheller RH: rab15, a novel low molecular weight GTP-binding protein specifically expressed in rat brain. J Biol Chem 267: 5768-5775, 1992.

23. Zuk PA and Elferink LA: Rab15 differentially regulates early endocytic trafficking. J Biol Chem 275: 26754-26764, 2000. 
24. Valencia A, Chardin P, Wittinghofer A and Sander C: The ras protein family: evolutionary tree and role of conserved amino acids. Biochemistry 30: 4637-4648, 1991.

25. Joshi S, Guleria RS, Pan J, Dipette D and Singh US: Heterogeneity in retinoic acid signaling in neuroblastomas: role of matrix metalloproteinases in retinoic acid-induced differentiation. Biochim Biophys Acta 1772: 1093-1102, 2007.

26. Cooper MJ, Steinberg SM, Chatten J, Evans AE and Israel MA: Plasticity of neuroblastoma tumor cells to differentiate along a fetal adrenal ganglionic lineage predicts for improved patient survival. J Clin Invest 90: 2402-2408, 1992.
27. Carlei F, Polak JM, Ceccamea A, et al: Neuronal and glial markers in tumours of neuroblastic origin. Virchows Archiv A Pathol Anat Histopathol 404: 313-324, 1984.

28. Echard A, Opdam FJ, de Leeuw HJ, et al: Alternative splicing of the human Rab6A gene generates two close but functionally different isoforms. Mol Biol Cell 11: 3819-3833, 2000.

29. Brauers A, Schürmann A, Massmann S, et al: Alternative mRNA splicing of the novel GTPase Rab28 generates isoforms with different C-termini. Eur J Biochem 237: 833-840, 1996.

30. Weinstein JL, Katzenstein HM and Cohn SL: Advances in the diagnosis and treatment of neuroblastoma. Oncologist 8: 278-292, 2003. 